vol. 29 - $n^{\circ} 4 \mid 2013$

Numéro ouvert

\title{
Forum Réfugiés, L'asile en France et en Europe. État des lieux 2010
}

\section{Maurice Blanc}

\section{(2) OpenEdition \\ 1 Journals}

Édition électronique

URL : https://journals.openedition.org/remi/6691

DOI : 10.4000/remi.6691

ISSN : $1777-5418$

Éditeur

Université de Poitiers

\section{Édition imprimée}

Date de publication : 1 décembre 2013

Pagination : 159-160

ISBN : 979-10-90426-10-8

ISSN : 0765-0752

\section{Référence électronique}

Maurice Blanc, "Forum Réfugiés, L'asile en France et en Europe. État des lieux 2010 », Revue européenne des migrations internationales [En ligne], vol. 29 - $n^{\circ} 4$ | 2013, mis en ligne le 01 décembre 2013, consulté le 14 avril 2022. URL : http://journals.openedition.org/remi/6691; DOI : https://doi.org/10.4000/remi. 6691 
Forum Réfugiés

L'asile en France et en Europe. État des lieux 2010. - Paris : La Dispute, 2010. - 386 p. (10 ${ }^{\text {ème }}$ rapport annuel)

ISBN : 978-2-84303-220-2

Forum Réfugiés est une association française créée en 1982. Elle participe à I'accueil des réfugiés et à la défense du droit d'asile. Convaincu que les problèmes des réfugiés appellent une réponse globale, Forum Réfugiés est membre du Conseil Européen pour les Réfugiés et les Exilés. Ce dixième rapport n'est pas le rapport d'activité de l'association mais, comme l'indique le sous-titre, un " état des lieux ". On pourrait le comparer aux rapports annuels de la Fondation Abbé Pierre sur l'état du mal logement en France. La publication de cet ouvrage a bénéficié du soutien du Fonds Européen pour les Réfugiés. II faut souligner l'effort de lisibilité qui a été fait dans l'écriture, l'iconographie et la mise en page, pour rendre la lecture de ce rapport facile et agréable.

Le plan en trois parties part du général pour aller vers le particulier : le monde, I'Europe, puis la France. Mais la réalité est plus complexe : ce n'est pas une logique vertueuse descendante, dans laquelle les principes généreux adoptés au niveau international se diffuseraient dans l'ensemble des nations. La logique ascendante (bottom up) l'emporte le plus souvent, mais elle n'a pas les vertus qu'on lui attribue naïvement. C'est ici l'effet " not in my backyard " (" les réfugiés, pas chez moi ! »). Les égoïsmes nationaux priment sur le principe de solidarité et le bien commun. Un communiqué du Parlement européen l'exprime ironiquement: " Les députés [européens] solidaires, les États-membres solitaires » (cité, p. 121).

La première partie est brève, mais elle donne des éléments de cadrage impor- tants, montrant que de nombreuses idées reçues sur les réfugiés sont sans fondement. Ainsi, $80 \%$ des réfugiés sont accueillis dans des pays pauvres (p. 9), proches de leur pays d'origine. Les pays dits du Nord, notamment la France et les États de I'Union européenne, ne sont pas les plus affectés. La crainte d'une invasion de la "forteresse Europe " par les réfugiés est un mythe (p. 61). La catégorie des " déplacés intérieurs " (à l'intérieur de leur propre pays) est sous-estimée et largement ignorée, alors qu'ils vivent dans des conditions particulièrement préoccupantes. Les catastrophes dites naturelles sont la cause d'un nombre croissant de déplacements forcés dans le monde (36 millions en 2008) ; mais ces déplacés ne sont pas considérés comme des réfugiés, alors qu'ils sont presque aussi nombreux que les déplacés à cause des conflits et de la violence (42 millions), et que les dérèglements du climat (qui ne sont pas purement " naturels ", risquent d'augmenter rapidement leur nombre (p. 15).

Si l'on prend l'Europe au sens géographique, la Tchétchénie et les pays du Caucase sont les principaux foyers de départ des réfugiés (p. 20). Dans le cadre de l'UE, les Balkans sont concernés, notamment les Roms qui sont discriminés et rejetés dans toute I'UE (p. 24). La mise en œuvre du droit d'asile dans I'UE est un travail de Pénélope, à recommencer chaque jour. La règle de l'unanimité pousse vers le bas le régime d'asile européen commun (p. 74). Le processus d'harmonisation est-il " en panne " ? (p. 81). Le rapport souligne l'ampleur des obstacles à surmonter, le plus important étant l'absence de volonté politique des États-membres de s'engager dans cette voie.

Pour la France, le rapport souligne quelques constats connus mais inquié- 
tants : les premières demandes d'asile sont en hausse et le réexamen des demandes rejetées est en baisse (p. 169). La procédure " prioritaire " pour accélérer le traitement des demandes réduit les droits des demandeurs (p. 191). La liste des " pays sûrs " est arbitraire et elle aboutit à des rejets dramatiques puisque, le pays étant réputé "sûr ", la demande d'asile est considérée comme infondée (p. 192). Un peu plus de la moitié des demandeurs d'asile sont déboutés (p. 222). Les aides au retour sont en baisse (p. 228), etc. Parmi les problèmes majeurs, le rapport souligne le traitement des mineurs étrangers isolés : ils sont abusivement traités comme des adultes et ils devraient bénéficier de l'aide d'un accompagnateur ; mais ce dernier est souvent désigné trop tard pour intervenir efficacement. Les Centres d'Accueil des Demandeurs d'Asile sont saturés (p. 248). Les problèmes de santé physique et mentale sont insuffisamment pris en compte (p. 269). L'obtention du statut de réfugié ne signifie pas la fin des problèmes. L'aide à leur insertion dans la société française se décompose entre un volet " intégration " qui relève de l'État central et un volet « insertion » relevant des collectivités territoriales, ce qui complique la coordination (p. 280). En 2002, Forum Réfugiés a mis en place dans la région Rhône-Alpes un programme "Accelair " d'accompagnement des réfugiés dans la recherche d'un emploi et d'un logement, ce qui implique une coordination renforcée entre les multiples partenaires.

Ce rapport est destiné à tous ceux qui veulent comprendre les enjeux du droit d'asile et de l'accueil des réfugiés. En même temps, il fournit aux praticiens et aux chercheurs une information précise et détaillée. Les annexes statistiques et la bibliographie spécialisée en font un outil de travail très précieux.

Maurice Blanc

Sociologue, professeur émérite, SAGE (Sociétés, Acteurs, Gouvernements, Europe) CNRS/Université de Strasbourg
Wihtol de Wenden, Catherine

La question migratoire au XXIe siècle.

Migrants, réfugiés et relations internationales. - Paris : Les Presses de Sciences Po, 2013. - 266 p. (Monde et sociétés)

ISBN : 978-2-7246-1284-4

Les migrations internationales sont pour les relations internationales et les sciences politiques, un objet d'étude très récent. Jusqu'au début des années 1990, les migrations étaient considérées comme un objet d'étude interne aux États, échappant de fait au champ des relations internationales. De même, les étrangers, parce que dépourvus du droit de vote, ont tenu une place très marginale dans la littérature politiste. Ce livre, à la fois dense et synthétique, se propose de mettre en évidence les conséquences que les nouvelles configurations migratoires ont pu avoir pour ces deux disciplines. À partir de la décennie 1990, le décloisonnement du monde qui a suivi la chute du mur de Berlin, la fin du régime international westphalien dans un contexte de globalisation, mais aussi le multiculturalisme, la montée en puissance du paradigme transnationaliste, les études comparatistes et le développement de l'interdisciplinarité sont autant de facteurs qui ont mis en avant la question migratoire.

Le livre s'arrête d'abord sur les migrations comme facteur de recomposition de la scène mondiale. Les nouvelles migrations sont le symptôme d'une interdépendance croissante entre les différents espaces mondiaux et entre les différents secteurs de l'activité humaine (politique, économique et sociale). Elles sont également les révélateurs de disparités démographiques, énergétiques, politiques et environnementales au niveau mondial. Elles ne se laissent plus appréhender par les anciennes catégories heuristiques. II faut dorénavant tenir compte de la diversité des causes de départ (crises économiques, politiques, environnementales), des itinéraires (migrations par étape, transit, retour, circulation bi ou multipo- 
laires, etc.), des motivations (stratégies familiales ou commerçantes, poursuite des études, émancipation politique, etc.) mais aussi de la porosité entre ces catégories : un même migrant peut être dans sa vie un réfugié, un clandestin, un travailleur, etc. De même une migration de transit peut devenir une immigration d'installation lorsque les frontières se ferment. Au-delà de ce mouvement brownien des hommes et des trajectoires se dessinent de nouvelles lignes de partage du monde. Aux anciens blocs Est/Ouest se substituent de nouvelles fractures : I'espace méditerranéen, la limite orientale de l'Union européenne, les frontières Mexique/États-Unis ou sud-africaines, etc. Et ce n'est pas le moindre des paradoxes de la mondialisation de faire de ces lignes de fractures les axes centraux de nouveaux "blocs " migratoires régionaux : le système USA Amérique latine, le système africain polarisé par l'Europe au Nord et l'Afrique du Sud pour sa moitié Sud, celui des pays du Golfe allant du Maroc à I'Asie du Sud, etc. Or cette partition du monde est appelée à durer. La force des réseaux sociaux, les nouvelles technologies de communications, la baisse continue des coûts de transports expliquent l'inertie contemporaine des flux migratoires.

Dans ces conditions, les migrations constituent pour l'État un défi pour sa souveraineté. L'analyse des relations entre migrations et souveraineté s'appuie sur un constat : le phénomène migratoire brouille les limites internes et externes de l'État. II déterritorialise les allégeances politiques et les identités. De fait, les études migratoires invitent à dépasser la conception wébérienne de l'État comme adéquation entre un appareil de pouvoir, une population et un territoire. Pour les États émetteurs, les diasporas deviennent un instrument d'externalisation de sa capacité d'action hors de son territoire. Depuis le milieu des années 1990, un nombre croissant d'États du Sud mettent en œuvre une politique diasporique dont les fins sont à la fois économiques (optimiser le volume et l'impact des transferts) et politiques (soutenir des causes politiques intérieures et extérieures). Pour les États récepteurs, les migrations soumettent la souveraineté à la pression des interdépendances globales. Dans ce contexte, la frontière devient à la fois un instrument et un enjeu stratégique. La frontière comme limite de territoire est un filtre définissant qui et ce qui peut la franchir. Mais la frontière est également intérieure (discriminations) et temporelle (durée des droits de séjour). Elle devient donc un objet polymorphe, à la fois spatial, juridique, sociologique et politique. Enfin, les politiques migratoires contemporaines donnent lieu à un nouveau type de diplomatie, tant au niveau bilatéral (accords de réadmission, externalisation des camps, codéveloppement), régional (UE, ALENA, etc.) que global (Forum Mondial Migrations et développement). Un réseau de nouvelles instances rassemble pays de départ et d'accueil, mais aussi organisations internationales (ONU, OIM, UNHCR, etc.) pour mettre en place une "gouvernance mondiale des migrations ". L'ouvrage évoque les particularités de ce nouveau jeu diplomatique. D'une part, on note la présence croissante d'acteurs non-étatiques : organisations internationales et non gouvernementales (humanitaires ou pro-immigration, associations de migrants) et prestataires de services privés (Frontex, sociétés de sécurité, service de gestion des visas). La seconde particularité est la délimitation de " champs migratoires d'influence ". Catherine Wihtol de Wenden entend par là un recoupement entre les systèmes migratoires et l'émergence de nouvelles ères d'expansion géopolitique dessinées par I'externalisation des politiques migratoires et des accords de développement. Ces ères peuvent être renforcées par d'anciens liens culturels (francophonie) et postcoloniaux.

Le dernier domaine qui a largement été affecté par les migrations contemporaines est celui de la citoyenneté. La particularité de la situation actuelle est 
celle d'une déconnexion croissante entre citoyenneté et nationalité. L'auteur rappelle que cette déconnexion n'est pas nouvelle. Tout national d'un État ne jouit pas des mêmes droits de citoyenneté (enfants, repris de justice) et une grande partie de la population des démocraties en a longtemps été exclue (femmes, pauvres, minorités ethniques ou religieuses, etc.). Inversement, pendant la Révolution, certains étrangers se sont vus attribuer le droit de vote sans être naturalisés (Thomas Paine). Aujourd'hui, les politiques d'intégration fondées sur l'inclusion politique et la lutte contre les discriminations, mais aussi la généralisation de la double nationalité ont permis l'extension du domaine des droits des étrangers dans les sociétés d'accueil et des nationaux expatriés dans les pays d'origine. Toutefois cette extension ne tend pas vers une égalité des droits entre étrangers et nationaux. Bien au contraire, on observe une multiplication des statuts de citoyenneté : du sans-papier dépourvu de tout droit à l'exception des droits humains les plus fondamentaux, au double national qui pratique une citoyenneté multi-nationale, en passant par les " denizens » qui jouissent de droits étendus au niveau local (droit de vote et parfois d'éligibilité aux élections locales) mais pas aux autres échelons de la vie publique. L'auteure relève que deux catégories sont largement occultées dans ce débat : les réfugiés d'une part et les apatrides de I'autre. Ces deux catégories n'ont cessé de voir le champ de leur droit se restreindre depuis deux décennies. Une nouvelle catégorie induite par la crise écologique, les réfugiés environnementaux, ne bénéficie, quant à elle, d'aucune reconnaissance. Le problème devrait toutefois prendre une acuité accrue dans les années à venir, avec la disparition sous les eaux des États insulaires du Pacifique (Tuvalu, Kiritbati).

L'ouvrage est une synthèse touffue des connaissances sur la question migratoire et sur ses interférences avec les grands thèmes de la science politique et des relations internationales : citoyen- neté, souveraineté, diplomatie, grandes lignes de partage du monde, etc. On peut regretter le manque de linéarité de son écriture (ce qui ne facilite pas la lecture d'un opus aussi densément fourni) et son manque d'ambition théorique. Par ailleurs, ce livre, initialement écrit en 2009, n'aborde pas la crise et ses conséquences sur les phénomènes décrits. Ce manque n'a pas été réparé ni dans la seconde édition du présent ouvrage, ni dans le dernier travail de l'auteure (Les nouvelles migrations. Lieux, hommes, politiques, Paris, Ellipses, 2013). Le chantier reste donc ouvert. Toutefois, "La question migratoire au XXle siècle " a le mérite de fournir une vision exhaustive des sujets actuels et à venir soulevés par les questions migratoires. Le livre plaide en faveur d'un " droit d'immigrer " en contrepoint d'un " droit d'émigrer " maintenant généralisé et pour une gestion concertée des flux migratoires. On peut espérer que ce livre permettra d'élargir la focale des deux disciplines qui restent, en leurs fondements, bien conservatrices.

Thomas Lacroix MIGRINTER CNRS/Université de Poitiers

\section{Laëthier, Maud \\ Être migrant et Haïtien en Guyane (Préface de Marie-José Jolivet). - Paris : Éditions du CTHS, 2011. - 319 p. (Le regard de l'ethno- logue)}

ISBN : 978-2-7355-0734-4

Maud Laëthier, anthropologue chargée de recherche à I'IRD, est spécialiste des migrations haïtiennes dans les Caraïbes. L'ouvrage dont il est ici question, issu de sa thèse de doctorat soutenue en 2007 , est une contribution significative à une meilleure compréhension du fait migratoire dans l'espace guyanais. Entre 1974 et 2006, la population recensée de ce territoire a plus que triplé, passant de moins de 60000 habitants à près de 200000 , cependant que la population immigrée s'élevait de 14 
à $30 \%$ de la population totale (p. 11). D'une part, cette situation migratoire a accentué le caractère multiculturel et pluriethnique de la société guyanaise, d'autre part, elle a nourri l'inquiétude des Créoles - élite sociale et politique de ce territoire - sur la place de leur identité dans la société guyanaise.

Dans ce contexte, le choix de Maud Laëthier de porter l'analyse sur la situation migratoire des Haïtiens en Guyane se justifie à plus d'un titre. D'abord, le fait migratoire est au cœur de l'identité haïtienne. Selon le ministère des Haïtiens vivant à l'étranger, créé en 1995 sous le gouvernement Aristide, Haïti comptait, en 2003, 2 millions d'émigrés sur une population totale de 8 millions. Ensuite, en Guyane, leur présence était estimée à 17000 personnes en 2006 , soit $30 \%$ de la population immigrée. Enfin, malgré son ancienneté - la mémoire des migrants haïtiens la fait remonter au milieu des années 1960 (p. 51) - et sa proximité culturelle avec les Créoles guyanais, cette présence haïtienne reste dévalorisée et stigmatisée par les Créoles.

Fondée sur une ethnologie à la fois participante auprès des migrants haïtiens et multi-située - en Guyane et en Haïti -, l'étude de Maud Laëthier se focalise sur les différentes constructions identitaires de ces migrants en Guyane dans leurs rapports avec leurs compatriotes (les " autres mêmes ") - migrants ou non - et avec les non-Haïtiens (les " autres différents »). Si I'on peut regretter quelques fois l'absence de données chiffrées permettant par exemple d'apprécier les similitudes et les différences entre les "nouveaux " migrants (ceux qui sont arrivés depuis les années 1990) et les actuels " anciens " migrants (ceux qui se sont installés au milieu des années 1960), l'auteure nous prévient que son " point de vue [est] délibérément qualitatif [...]. Les études de cas se [voulant] éclairantes sans être représentatives $»($ p. 16).

\section{Les " autres mêmes "}

Pour décrire les processus de reproductions et de transformations des constructions identitaires des migrants haïtiens en Guyane, Maud Laëthier expose d'abord les modalités de la migration d'Haïti vers la Guyane. Elle s'attache alors à mettre en évidence les éléments d'une certaine forme d'autonomisation du mouvement migratoire des Haïtiens. Parmi ses éléments en Haïti, elle relève les multiples agences de voyage, qui permettent de voyager sous une fausse identité avec des passeports et des visas légaux (p. 65), et les réseaux de prêteurs professionnels, qui peuvent appliquer parfois des taux de remboursement exorbitants de $100 \%$ (p. 68). Surtout, elle montre l'importance dans la réalisation du projet migratoire des réseaux de parenté, de voisinage et d'interconnaissance, qui reposent sur la solidarité, la compréhension de la nécessité de partir " chercher la vie » (p. 53) ailleurs et l'espoir que le migrant, membre désormais de la " diaspora " haïtienne (p. 70), démontrera ses capacités financières à aider ceux qui sont restés.

Cette description de l'autonomisation des mouvements migratoires haïtiens permet à l'auteure ensuite d'aborder la construction juridique et politique des identités des migrants haïtiens en Guyane. Cette dernière s'appuie sur des récits d'expériences de l'illégalité dans un contexte guyanais de législation d'exception, comme en témoigne l'impossibilité du recours suspensif lors d'une reconduite à la frontière (p. 84). Cette législation tend ainsi à produire davantage d'irrégularité, laquelle entrave le processus d'intégration socioéconomique des migrants haïtiens et, ce faisant, renforce leur identité communautaire et leur image dévalorisée dans la société créole guyanaise.

Cette image stigmatisée de la présence haïtienne en Guyane qui perdure, malgré son ancienneté, procède également de la construction d'un espace de relations 
communautaires des Haïtiens, au sein de ce qui est perçu en Guyane comme un quartier spécifiquement haïtien. Dans ce cadre, Maud Laëthier s'appuie sur une enquête menée dans la cité Boutezelle, située dans la banlieue est de Cayenne, où domine l'habitat informel et se développe une organisation sociale haïtienne fondée sur un système d'entraide quotidien et la pratique d'activités reconnues comme spécifiquement haïtiennes, tel le jeu de loterie la "borlette " (pp. 132-133). Elle se caractérise par le partage d'une cour, le "lakou " (pp. 119-120), au sein d'une unité de résidence, définie comme un ensemble de maisons mitoyennes rassemblant les membres d'une même famille et des voisins désignés par les termes de " cousin " et de " ma commère " (pp. 126-127), pour indiquer une proximité amicale et géographique. Mais cette organisation sociale dans les quartiers haïtiens de Guyane n'est pas une reproduction à l'identique de l'organisation sociale en Haïti. Par exemple, le " lakou " d'Haïti est modifié en Guyane, en ce sens qu'il ne regroupe plus uniquement des parents, mais aussi des interconnaissances, le plus souvent en raison d'une même commune d'origine.

La stigmatisation de la présence haïtienne en Guyane par les Créoles engendre une construction identitaire communautaire, qui participe au processus d'ethnicisation de la société guyanaise. Elle doit être analysée en lien avec une politique migratoire spécifique en Guyane, qui conduit les Haïtiens à " chercher la vie " dans l'irrégularité et, ce faisant, à produire leur propre espace de vie et d'entraide. De plus, elle assigne aux Haïtiens l'image du pauvre migrant acceptant des emplois perçus comme avilissants ; assignation identitaire que bien souvent les migrants haïtiens ne remettent pas en cause. Pour autant, la situation migratoire de ces migrants en Guyane offre également l'opportunité de former et d'entretenir un " nous " haïtien, fondé sur une identité haïtienne construite en Guyane tant par l'apport des migrants eux-mêmes que par les relations avec les différentes composantes de la société guyanaise.

\section{Les " autres différents "}

II existe, au sein de la population haïtienne, des intermédiaires entre la société locale et les migrants haïtiens. Ces intermédiaires, souvent scolarisés pendant plus de dix années (p. 230) et naturalisés (p. 240), forment l'élite de la communauté haïtienne et témoignent en creux des mobilités sociales ascendantes ayant cours au sein de la population migrante haïtienne. Appelés "gwo nèg " (p. 212), ils peuvent être des pasteurs des assemblées religieuses protestantes ou des responsables des associations culturelles haïtiennes, qui se sont développées en Guyane. Ils sont également les acteurs d'une seconde configuration étudiée par Maud Laëthier, au cours de laquelle peut s'opérer un glissement identitaire de l'état " d'autre stigmatisé " à celui " d'autre semblable ".

À travers la description de l'engagement des Haïtiens dans des institutions communautaires (les Églises protestantes et les associations), l'auteure met en relief leur volonté de s'ouvrir aux autres groupes communautaires en Guyane - en fait surtout au groupe créole - et partant, de s'intégrer à la société guyanaise. Cet aspect constitue un des apports majeurs de l'ouvrage, en ce sens que cette volonté révèle, en effet, que l'intégration reste I'horizon d'attente des migrants haïtiens : I'engagement dans les institutions communautaires souligne comment I'appartenance communautaire peut être transcendée par la volonté d'intégration, au nom d'une universalité religieuse ou sociale. Ainsi, Maud Laëthier montre bien l'évolution, entre 1960 et les années 2000, des actions des associations haïtiennes : d'abord tournées vers le pays d'origine, notamment les communes du Sud, d'où proviennent majoritairement les migrants haïtiens, leurs actions s'orientent de plus 
en plus à destination de la " communauté haïtienne guyanaise " (pp. 229-230). Dès lors, les premières activités de ces associations ne sont pas la promotion d'une identité haïtienne mais bien l'alphabétisation, le soutien scolaire et la formation professionnelle des Haïtiens en Guyane, en vue de réaliser l'intégration de leurs compatriotes (p. 225).

Mais cet engagement dans ces institutions communautaires repose sur la définition d'une auto-identification haïtienne, dans laquelle seraient mises à distance les pratiques haïtiennes les plus stigmatisées, telle celle du vaudou, pourtant au cœur de ce que les autres " groupes communautaires " perçoivent comme des pratiques haïtiennes " authentiques ». Or c'est bien la pratique du vaudou qui devient le vecteur d'intégration, par un renversement du stigmate, comme le démontre Maud Laëthier dans la dernière configuration identitaire analysée. Celle-ci s'appuie sur l'étude de cas d'un migrant arrivé en 1982, qui d'abord maçon, devient prêtre vaudou (ougan) et épouse une Créole (p. 252). Depuis une dizaine d'années, cet ougan initie aux pratiques de son culte, en Guyane et en Haïti, ses compatriotes et des Créoles guyanais et antillais. C'est ainsi que, par la valorisation du vaudou et l'affirmation de l'authenticité de son enseignement, ce migrant haïtien a pu à la fois construire son ascension sociale et son intégration dans la société guyanaise.

Au total, Etre migrant et Haïtien en Guyane est un ouvrage novateur dans le domaine des migrations, en ce qu'il met en lumière un double mouvement, trop souvent perçu comme contradictoire. D'une part la situation migratoire des Haïtiens conduit à un fort mouvement de communautarisme dans le contexte guyanais, d'autre part ce communautarisme, loin de provoquer un repli sur soi, peut être une ressource dans les efforts des migrants pour devenir membre de la société guyanaise, horizon d'attente de la grande majorité des migrants ${ }^{1}$. De ce point de vue, cet ouvrage nous invite notamment à poursuivre la réflexion sur la notion de multiculturalisme au sein de la république.

Maurice Edenz Doctorant en Histoire École doctorale de Sciences Po
1 À propos des facteurs qui freinent ou facilitent I'intégration des immigrants aux États-Unis, voir Eva Morawska, Sociology of immigration: (Re)Making Multifaced America, New-York, Palgrave Macmillan, 2009. 